\title{
Metamizole Sodium Induces Neural Tube Defects in a Chick Embryo Model
}

\author{
Yahya GUVENC ${ }^{1}$, Deniz BILLUR ${ }^{2}$, Sevim AYDIN² ${ }^{2}$ Ersin OZEREN ${ }^{3}$, Adnan DEMIRCI $^{4}$, Fatih ALAGOZ ${ }^{5}$, \\ Ali DALGIC 5 , Deniz BELEN ${ }^{5}$ \\ ${ }^{1}$ Dr. N. K. Sincan State Hospital, Department of Neurosurgery, Ankara, Turkey \\ ${ }^{2}$ Ankara University, School of Medicine, Department of Histology-Embryology, Ankara, Turkey \\ ${ }^{3}$ Aksaray State Hospital, Department of Neurosurgery, Aksaray, Turkey \\ ${ }^{4}$ Yalova Hospital, Department of Neurosurgery, Yalova, Turkey \\ ${ }^{5}$ Ankara Numune Traning and Education Hospital, Department of Neurosurgery, Ankara, Turkey
}

\section{ABSTRACT}

AIM: The aim of this study was to investigate the effects of metamizole sodium on neural tube development in the early stage chick embryo model that complies with the first month of embryonic development in mammals.

MATERIAL and METHODS: A total of 40 fertilized chicken eggs were divided into 4 equal groups. The eggs were incubated in the incubator at a temperature of $37.8 \pm 2^{\circ} \mathrm{C}$ with $60 \pm 5 \%$ humidity. Group A was the control, Group B was administered physiological saline, Group C was administered $30 \mathrm{mg} / \mathrm{kg}$ metamizole sodium (based on the therapeutic index range of it used in humans) and Group D was administered $90 \mathrm{mg} / \mathrm{kg}$ metamizole sodium. All embryos were removed from the egg at the $48^{\text {th }}$ hour and morphologically and histologically examined.

RESULTS: Normal development was seen and the neural tube was closed in 17 embryos in Groups $A$ and $B$. A neural tube defect was seen in 2 embryos in group $A$ and in 1 embryo in group B. A neural tube closure defect was seen in all embryos in group $C$ and 9 embryos in group D. There was 1 dead embryo in Group D.

CONCLUSION: Metamizole sodium was seen to produce a neural tube defect in the chicken embyro model.

KEYWORDS: Metamizole sodium, Neural tube defects, Chick embryo

\section{INTRODUCTION}

Congenital anomalies are changes in the natural anatomic structure present at birth. Congenital central nervous system anomalies are the most common, followed by congenital cardiovascular abnormalities (9). Neural tube defects (NTD) are heterogeneous and complex congenital anomalies of the central nervous system. The worldwide incidence of neural tube defects varies between $0.1 \%$ and $1 \%$ (3).

The causes of congenital abnormalities are multifactorial. Although the cause of the malformation is not known in up to $65-75 \%$, these cases are associated with genetic factors in
$25 \%$, environmental reasons in $10 \%$, chromosome aberrations in $3 \%$ and factors such as drugs, infection and radiation exposure during pregnancy in about 3\% (10).

Metamizole sodium was synthesized in 1920 and production started in Germany in 1922. Metamizole sodium (dipyrone) is a non-opioid analgesic drug used commonly in many countries and also has an antipyretic effect. Metamizole side effects are nausea, vomiting, diarrhea, headache, dizziness, hepatic dysfunction, renal dysfunction, skin reactions (rash, urticaria, erythema), increase in bronchial asthma attacks, anaphylactic shock, agranulocytosis and aplastic anemia 
(15). The use of metamizole sodium during pregnancy is classified as pregnancy category $\mathrm{C}$. There is little information on precautions regarding metamizole use during pregnancy and its safety (14). It is reported in the package insert of the drug that it passes the placenta but there is no evidence that it is harmful to the fetus. It does not show any teratogenic effect in rats or rabbits.

We investigated the effects of metamizole sodium on the neural tube at the early-stage chicken embryo model that complies with the first month of the embryonic development of the mammalian spine.

\section{MATERIAL and METHODS}

A total of 40 fertilized chicken eggs of the Atabey ${ }^{\circledR}$ kind (Republic of Turkey Ministry of Agriculture, Ankara Poultry Institute) and free of specific pathogens were used for this study. The eggs were placed in the incubator in order to ensure the continuity of the embryos and to have them available at the times desired. The incubator was kept at a constant temperature range of $37.8 \pm 0.2^{\circ} \mathrm{C}$ and humidity of around $60-70 \%$. The eggs were randomly divided into 4 groups. Group A was incubated for 48 hours without any additional procedure. The other eggs were opened with the windowing method at the $28^{\text {th }}$ hour of incubation ( $8^{\text {th }}$ stage according to the Hamburger-Hamilton staging). Physiological saline solution and metamizole sodium (Novalgin, Sanofi Aventis llaclari - Istanbul) were administered to the embryo with the in ovo method within a volume of $10 \mu \mathrm{L}$. Group B was only administered physiological saline. Group $C$ was administered $1.8 \mathrm{mg}$ metamizole sodium based on the drug's human therapeutic index (30 mg/kg). Group D was administered 5.4 $\mathrm{mg}$ metamizole sodium based on three times the dose $(90 \mathrm{mg} /$ $\mathrm{kg}$ ) according to the therapeutic index of metamizole sodium. The embryos were removed from the egg at the $48^{\text {th }}$ hour (12th stage according to the Hamburger-Hamilton staging).

All embryos were fixed with $10 \%$ buffered formalin for 48 hours. The neural tubes were examined under a stereomicroscope
(Nikon, SZX 1000) to observe gross anatomy, and photographs were taken. Routine histological processing was used for the light microscopic examination of the embryos. After washing under tap water, dehydration was performed through graded alcohol series. The tissues were then made transparent with xylene and embedded into paraffin. Transverse serial sections $4 \mu \mathrm{m}$ thick were obtained from the paraffin blocks with the Leica RM 2125RT microtome and stained with HematoxylinEosin (H-E). The preparations were evaluated using the Zeiss Axio Scope A1 light microscope by two histologists blinded to the groups and photographs were taken.

\section{RESULTS}

Group A: There were 16 somites and the neural tube was closed, stage 12 of the Hamburger-Hamilton classification, in 8 of the 10 chick embryos in the group (Figure 1A) while the neural tube was not closed in the other two embryos under the stereomicroscope. The tissue samples observed under the light microscope after staining with $\mathrm{H}-\mathrm{E}$ were found to be consistent with the stereomicroscopic examination (Figure 1B).

Group B: We found that 9 of the 10 chick embryos administered physiological saline were at the expected embryonic stage according to the Hamburger-Hamilton classification. The neural tube was not closed in one embryo on stereoscopic examination. Light microscope findings were consistent with the stereomicroscope.

Group C: The neural tube in all of the 10 chick embryos was not closed with the dose of metamizole sodium ( $30 \mathrm{mg} / \mathrm{kg} /$ day) used within the therapeutic index (Figure 2), and there was developmental retardation present in one embryo according to the Hamburger-Hamilton classification with fewer somites than expected (Figure 3).

Group D: One of the chick embryos died after the administration of metamizole sodium at 3 times the therapeutic index dose (90 $\mathrm{mg} / \mathrm{kg} /$ day) and the neural tube was not closed in nine
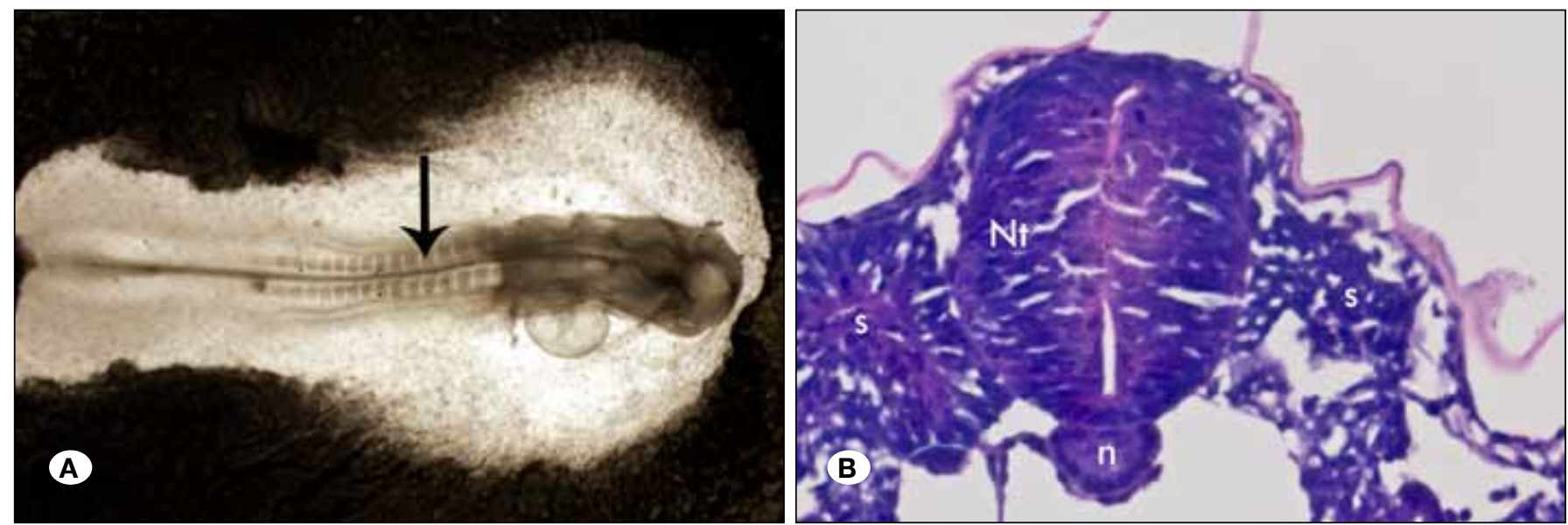

Figure 1: The neural tube is observed to be closed in the stereomicroscope image belonging to group $A(A)$. $A$ section was obtained at the level indicated by the arrow in Figure $1 \mathrm{~A}$ and stained with $\mathrm{H}-\mathrm{E}$. The neural tube is seen to be closed (B) (H-E, X40). Nt; neural tube, n; notochord, s; somites. 
embryos (Figure 4 A,B). Developmental retardation was present in three embryos.

\section{- DISCUSSION}

The primitive line, notochord and neural tube begin to develop from the 3rd week of the embryo. The spinal cord is formed with the development of the caudal part of the 4th pair of somites of the neural tube. Exposure to teratogens during this period of the pregnancy affects the development of neural tissue in the embryo and leads to developmental retardation of the neural tube (13) and its reopening after being formed or to neural tube rupture (6).

Metamizole sodium manufacture started in 1922. It was one of the most common analgesic and antipyretic drugs worldwide. It was then found to cause agranulocytosis and fatal complications in 1970. Its use was forbidden in Switzerland in 1974, the USA in 1977 and in close to 30 other countries including Japan, Australia, Iran and some European Union countries in subsequent years. It is still a popular analgesic in some European (Germany, Russia, Poland) and other countries (Turkey, Brazil, Mexico, Israel, Egypt) and it is known to be used without a prescription in some countries.

Metamizole sodium is a drug with an analgesic and antipyretic effect but no anti-inflammatory component. It decreases prostaglandin biosynthesis by inhibiting cyclooxygenase-1 and cyclooxygenase-2 in the central nervous system (7). It is thought to show its analgesic effect by decreasing prostaglandin synthesis through cyclooxygenase-3 enzyme inhibition in the spinal cord posterior horn $(1,11)$. The molecular basis of neurulation is largely unknown. The embryo becomes less resistant to challenge by environmental stress when neurulation occurs. We think metamizole sodium may create stress in the embryo and block neurulation but the mechanism is unknown and further studies are needed.

Pregnant women who use metamizole can experience urticaria, hypotension, bronchospasm, and cardiac arrhythmia as side effects but the most severe ones are agranulocytosis and anaphylactic reactions. Agranulocytosis and anaphylactic

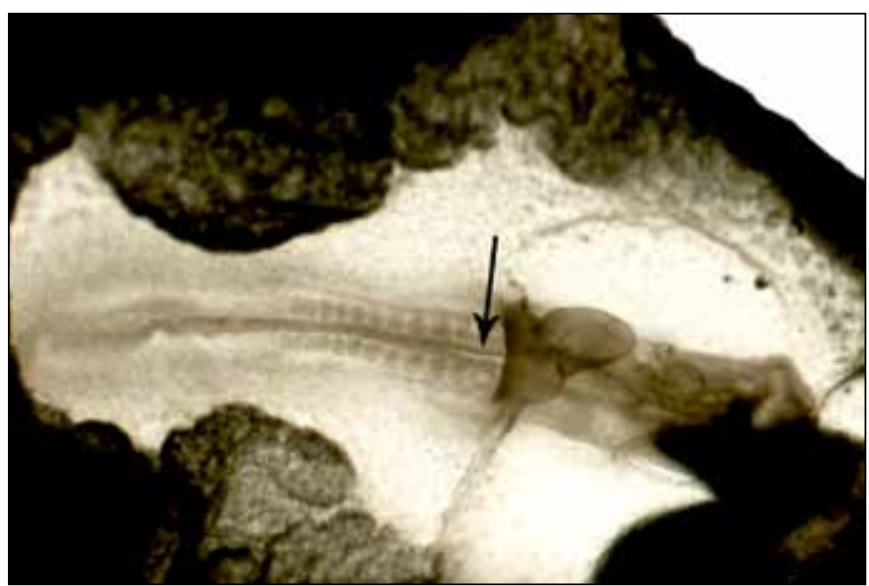

Figure 2: The opening in the neural tube (arrow) is seen in the stereomicroscope view of Group C.

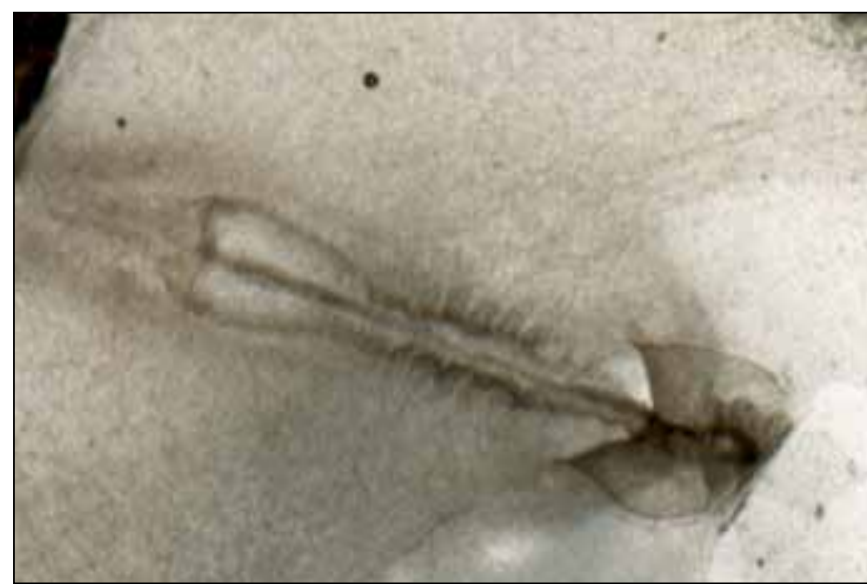

Figure 3: Developmental retardation seen in the stereomicroscope view of Group C.
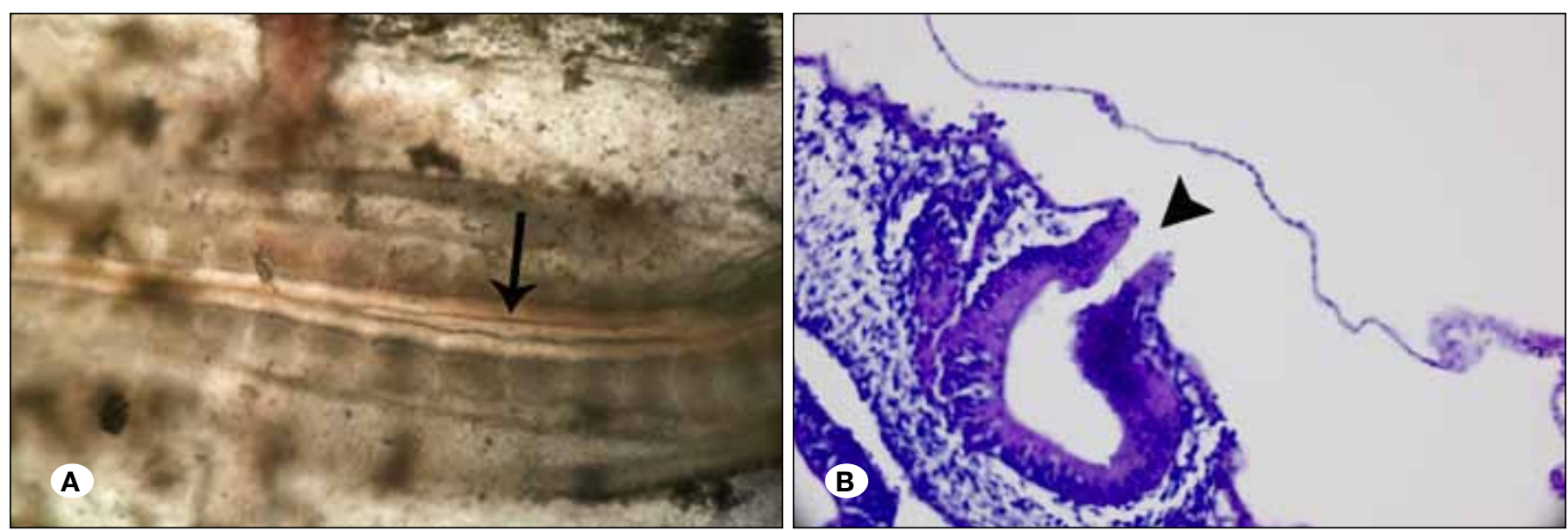

Figure 4: The neural tube is not closed (arrow) in the stereoscopic image of the group D embryo that was administered metamizole sodium at a dose exceeding the limits of the therapeutic index (A). The neural tube is open (arrow) in the light microscope image of the same group (B) (H-E, X20). 
shock can be fatal. Some studies have investigated the connection between the use of metamizole sodium during pregnancy and the development of congenital anomalies. Administration of metamizole sodium to pregnant rats and evaluation of transplacental passage and carcinogenicity has shown no carcinogenicity (8). Da Silva Dal Pizzol et al. investigated the presence of congenital anomalies, intrauterine death, preterm birth, and low birth weight in pregnant women who used metamizole sodium and found no relationship (4). Bar-Oz et al. compared 108 pregnant women who used metamizole sodium in the first trimester of pregnancy with pregnant women who used acetaminophen as a control group. Both groups were evaluated for birth weight and gestational age of the child, live birth rate, and spontaneous abortions. No significant difference was found between the incidence of major malformation in patients using metamizole (3\%) and the control group (2\%) (2). A weak connection was detected between metamizole sodium use during pregnancy and Wilms tumor in the child (12). Weintraub et al. reported oligohydramnios and ductus arterious stenosis in the fetus following metamizole sodium use in pregnant woman (14). Some articles report the development of acute renal failure after the use of metamizole sodium in pregnant women (5). It is clear from these articles that safety information regarding the use of metamizole sodium in pregnancy is inadequate. Its use in humans during pregnancy without more detailed investigations may not be as safe as it is believed. We believe that every effect of this drug that is used widely across the world should be studied in detail.

We did not come across a comprehensive article on the effects of metamizole sodium on neural tube development with a literature search. The neural tubes of the chick embryos were seen not to be closed with some embryos showing developmental retardation with a small number of somite formation even within the therapeutic dose range of metamizole sodium. Metamizole sodium administered at high doses was found to cause a neural tube defect, developmental retardation, and even death in the chick embryo. Metamizole sodium was therefore shown to cause a neural tube developmental defect in the chick embryo with teratogenic effects in this study. This study may set an example for future studies on the possible effect of metamizole sodium on the formation of neural tube defects in humans. Further studies on this matter are obviously needed in vivo, in vitro, and at the molecular level.

\section{REFERENCES}

1. Arellano F, Sacristan JA: Metamizole reassessment of its therapeutic role. Eur J Clin Pharmacol 38: 617-619, 1990

2. Bar-Oz B, Clementi M, Di Giantonio E, Greenberg R, Beer M, Merlob P, Arnon J, Ornoy A, Zimmerman DM, Berkovitch M: Metamizol (dipyrone, optalgin) in pregnancy, is it safe? A prospective comparative study. Eur J Obstet Gynecol Reprod Biol 119(2):176-179, 2005

3. Botto LD, Moore CA, Khoury MJ, Erickson JD: Neural tube defects. N Engl J Med 341:1509-1519, 1999

4. Da Silva Dal Pizzol T, Schüler-Faccini L, Mengue SS, Fischer MI: Dipyrone use during pregnancy and adverse perinatal events. Arch Gynecol Obstet 279(3):293-297, 2009

5. De la Nieta S, Rivera F, De la Torre M, Roberto Alcázar R, Caparrós G, Paz Alcaide M, Vozmediano C, Sánchez A: Acute renal failure and oligohydramnios induced by magnesium Dipyrone (metamizol) in a pregnant women. Nephrol Dial Transplant 18(8):1679-1680, 2003

6. Gardner WJ: Myelomeningocele the result of rupture of the embryonic neural tube. Cleve Clin Q 27:88, 1960

7. Hinz B, Cheremina O, Buchmakov J, Renner B: Dipyrone elicits substantial inhibition of peripheral cyclooxygenases in humans: New insights into the pharmacology of an old analgesic; FASEB J 21:2343-2351, 2007

8. Kramer M: Chronic toxicity of pyrazolones: The problem of nitrosation. Br J Clin Pharmacol 10 Suppl 2:313S-317S,1980

9. Manning $\mathrm{N}$, Archer $\mathrm{N}$ : Treatment and outcome of serious structural congenital heart disease. Semin Neonatol 6:37-47, 2001

10. Ornoy A, Arnon J: Clinical teratology. West J Med 159(3):382390, 1993

11. Pierre SC, Schmidt R, Brennels $C$, Michaelis M: Inhibition of Cyclooxygenanses by Dipyrone. Br J Pharmacol 151:494503, 2007

12. Sharpe CR, Franco EL: Use of dipyrone during pregnancy and risk of Wilms' tumor. Brazilian Wilms' Tumor Study Group. Epidemiology 7(5):533-535, 1996

13. Von Renklinghausen $\mathrm{E}$ : Untersuchungen über die spina bifida, Arch Pathol Anat 105:243-373,1886

14. Weintraub A, Mankutz D: MHA dipryone-induced oligohydramnios and Ductus Arteriosus Restriction. IMAJ 8:722-723, 2006

15. Żukowski M, Kotfis K: Safety of metamizol and paracetamol for acute pain treatment. Anestezjol Intens Ter 41(3):170-175, 2009 